\title{
Critical Evaluation on WSNs Positioning Methods
}

\author{
Putri Kevin', and Dian Viely ${ }^{2}$ \\ ${ }^{1,2}$ Department of Computer Science, Brahijaya University, Indonesia \\ Correspondence should be addressed to Putri Kevin: lettylord@yahoo.com
}

Copyright (C) 2021 Made Putri Kevin et al. This is an open access article distributed under the Creative Commons Attribution License, which permits unrestricted use, distribution, and reproduction in any medium, provided the original work is properly cited.

\begin{abstract}
WSN gained a lot of attention because they are small and economical devices with low power utilization, and finite computing resources are progressively being enfolded in various application situations, including environmental monitoring, reconnaissance tracking, and health monitoring. In many applications of this type, node localization is an intrinsic parameter of the system. To report events origin, routing, and network coverage (A\&Q), assist group querying of the sensors Localization process is mandatory. Localization is categorized into two types, which are a range-based or range-free scheme which is further divided into two sub-schemes as a fully and hybrid. For the detailed analysis and study, we are going to investigate localization schemes based on static and mobile WSN in this work. This research opens the new paradigm and future planning for the localization algorithms mainly how sensors are deployed? What kind of measures are taken into account to boost the algorithms so that the system can calibrate itself in changing environments?
\end{abstract}

KEYWORDS- RSSI, localization, anchor nodes, wireless sensor networks, mobile computing

\section{INTRODUCTION}

MEMS IC technology is drastically progressing now a day along with wireless communication. Connecting multiples sensors to a network from WSN[1]. The sensor reads the physical characteristic like temperature, pressure, and distance, etc. WSN helps in health monitoring of patient health, military targets with location recognition. In localization, collected data from the sensor node is useless if the location is not precise. To fix this localization problem, equip each sensor node with GPS, which is practically impossible because of cost and power consumption increase [2].GPS glitch in dense forest, underground and indoor. Self-localization method sensor node used localization protocol to estimate position. Beacon nodes are used to estimate location in this protocol. Blind nodes provide information about the position of non-beacon nodes, known as a blind node. Blind nodes use information messages of beacon to locate his position[3-6].

A complete localization algorithm consists of two parts one is to compute the distance and angle approximation and the other is location computation. The distance or angle between the sensor nodes are computed through Time difference of arrival (TDoA), Time of arrival (ToA), RSSI and Angle of arrival (AOA). Hop count is also a main technique to compute the distance and angle between the nodes. The available information like RSSI also helps to compute the angle position between nodes and reference node [7-11]. For this the most common techniques are lateration, triangulation, bounding box, probabilistic approach, and fingerprinting. The classification of position techniques is presented in Section 2, while a detail study of localization algorithms are presented in Section 3 and Section 4 conclude the paper with future direction.

\section{CLASSIFICATION OF LOCALIZATION SCHEMES}

Range-measurement technique is a well-known parameter used in range-based algorithm's while it's not available in Range free localization algorithms. The location of node this information or topology is used i.e.: who is within the communication range of whom[12].

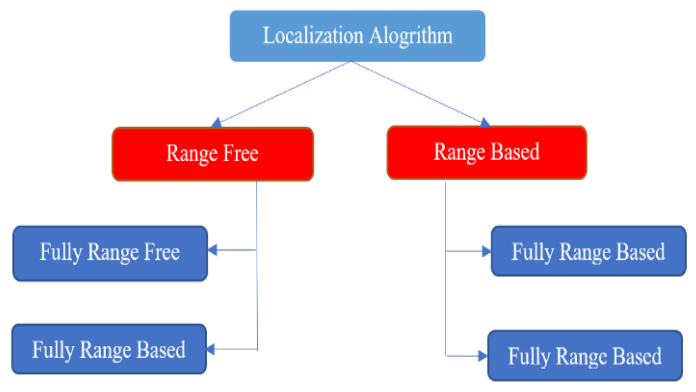

Fig. 1: Classification of localization algorithm.

Range free localization algorithms The range-free localization algorithms help to find out the location of an unknown node. The range-free localization algorithms extend into two more types: Hybrid range free and full range free. An entirely free localization algorithm uses the principle method of information topology and connectivity.

\section{A. Anchor based approaches}

The fully range-based localization on the anchor node has a range of techniques, which includes Centroid[13], Dv Hop[14], and APIT[15].The most common technique is the CA. The CA comprises location information, uses anchor nodes to estimate node location.CA method implementation is easy, economical, and straightforward. This method is limited to 2D networks [11].Niculescu and Nath propose Dv-Hop [16]; this method depends upon the vector distance exchange method. Dv-Hop was implemented in three steps. Step 1, Anchor node transmits data to its adjacent neighbors. All anchor nodes get a minimum hop count from every anchor node[8]. Step 2, The information from step 1 is used in calculating the average size of the hop count for 
the anchor node. Step 3. When the unknown node obtains three or more distances among the anchor nodes, an unknown node position can be estimated using the trilateral approach[17]. The DV-Hop method is simple, easy to implement, not dependent on range measurement error, and has a bad localization accuracy. APIT[18], Approximate point in triangulation in which three nodes are connected into a triangular pattern. This algorithm is less affected by an environmental factor, low hardware requires. The accuracy of APIT is mainly affected by node presence with a triangular pattern or not. In APIT, more anchor point than average is needed in localization. We found that the APIT algorithm does not assume the correlation between absolute distance and radio signal strength, so we consider it an entirely free Algorithm. ADAL[19] use mobile beacons with a rotary directional antenna to send a message in a calculated azimuth consistently; an unknown node uses the CA convergence area of beacon message at its location[20].

\section{B. Anchor free approaches}

MDS-MAP is a multidimensional scaling map, which is a centralized range free algorithm.MDS-MAP forms a relative map (relative position of sensor node w.r.t anchor node)[21]. MDS-MAP produces the most precise localization information. The main disadvantage of MDS is high time complexity, large bandwidth required, and computation requires when a large no of sensors are innetwork.

\section{Hybrid range-free localziation algorithms}

These algorithms are a combination of different methods based on topology and connection. Iterated Hybrid Localization Algorithm (IHLA) establish on CA and DV-Hop method [22]. Using the centroid method, the unknown node determines the coordinates. It reassesses the distances between beacon and unknown nodes based on the DV-Hop method. This method is more accurate but complex and requires high computational time. Range free implementation is easy, where location accuracy is not critical. Low cost doesn't require new hardware; location error depends on the network's complexity. The hybrid takes an advantage over range free because a different method combines for better performances.

\section{Range-based localization algorithm}

The range-based algorithm uses distance measurement methods. This method is further divided into two types, Fully Range-based and Hybrid Range based. The fully range-based localization algorithm is further divided into Anchor based and hybrid approaches, which discuss in detail below.

\section{E. Anchor based approaches}

RSSI suggested method in [23] is based on the estimated distance between the sensor node and RSSI measurement. The signal strength is inversely proportional to the distance between the sensor node. The radio model is used to convert radio signal strength into the distance[24]. The RSSI is sensitive to noise, obstacles, and environmental factors[25]. The error due to multipath-propagation fading power and reflection should be in an account in measured value [26]. RSS is the most common method, cheap and simplest techniques. RSS requires more data, which reduces the sensor lifetime. To extend the sensor's life span, an ILOR ZigBee based approach is used[27]. AOA angle of arrival proposed in [28], two algorithm DV-radial and DvBearing were proposed [29]. Complexity-reduced 3D trilateration Localization Approach (COLA)[30] based on RSSI values simplifies the positioning process by decreasing 3D computation to 2D computation. The AOA capability allows for each node bearing to the adj acent node with respect to the node's axis. The angle at which an object can be seen from another point is a radial. In [19], the ALAR method is used; a large antenna is required for a high SNR ratio. This method is accurate but limited by the antenna's direction due to the multipath, reflection, and shadowing effect. The main disadvantage is cost due to additional hardware. Time of arrival (TOA) is also a known time of flight (TOF)[31]. The distance between receiver and transmitter is calculated by multiplying the signal's propagation time with signal speed. GPS used these technologies. TOA is required to have a synchronized transmitter and receiver. Synchronization adds complexity and cost[32]. Time difference of arrival (TDOA)[33] in WSN are obtained by two methods. From unknown node to two anchor node TAO can be measured and calculated time difference. In a second way, two transmission mediums of very different propagation speed are accessible, that's mean two different signals, for example, ultrasound/ acoustic and radio signals. When two different signals are transmitted simultaneously, and the time delay is equal to zero, the estimated distance between the transmitter and receiver can be calculated by the given formula. This system's main disadvantage is that additional hardware is required, and ultrasound signals can be stopped diverge by an obstacle.

\section{F. Anchor free approaches}

Assumption based coordinate algorithm (ABC)[34] is centered on RSSI measurement to calculate the distance between nodes. To accommodate inter-node distances, this schematic is the first to select four nodes and.ABC algorithm is simple but poor in location accuracy, and calculation is not complex. In addition, if measurement are distorted by noise, the algorithm can lead to incorrect nodes. In [35], Moore proposed the robust distribution network localization (RODL), in which the node measures(Noisy Range) the distance to the adjacent node by using TDOA for locating a node in a sensor network, how noise calculation induces incorrect displacement. In this method, each node becomes a cluster of the center and calculates its relative position, which can be entirely localized. The beauty of this scheme is that it cant use any external hardware and anchor node while localization. Without using any anchor node, this method effectively localized the node in a sensor network.

\section{G. Hybrid Range-based localization algorithm}

In ATPA [35], is a based on AOA and TOA scheme which effectively measure the localization of a sensor node. This technique measures the node position in 3 different ways. AoA is used to measure the angle between the nodes and ToA is used to measure the time since all nodes arrival time is different than other. For position computation the geometric interpretation is used and applied to partial filter. [26]. Last, fuzzy adoptive control helps to adjust the localization computation [36]. Bishop in[28] proposed a method that is a hybrid technique of AOA and TDOA. This[37] proposed system is genetically more robust. In 
[38], the fusion of RSSI and TDOA (FRTL) is proposed for indoor localization and better robust. TDOA is a primary distance estimation localization approach that can collect and train RSSI data (For known distance ); when TDOA is unavailable, the training data used for missing is unavailable in the presence of acoustic noise TDOA estimation[29]. FRTL is in terms of distance computation, and localization is better.

An HRTL is a hybrid TOA/ RSSI algorithm proposed in[39] which used both TOA and RSSI computation without LOC and NLOS and used the hypothesis test [30].

Blumenthal proposed WCA (Weighted Centroid Algorithm)[40]. This algorithm used RSSI as a measurement of localization as a centroid scheme. Anchor node keep transmitting packets and choose larger RSSI for data matrix. An unknown node calculates the anchor node weight, using acquired RSSI measurement to estimate the location. This algorithm increases the performance of localization in centroid with low energy usage and external hardware.

In [41], the RSSI-based DV-Hop algorithm is proposed in which an unknown node one hop away from the anchor point uses RSSI computation to estimate the distance between its adjacent neighbor and anchor node. Unknown node locates itself using triangulation methods. This algorithm is better than DV-Hop but limited to the anchor node getting RSSI calculation. In [42], Magnani proposed SSFL that fuses AOA and TDOA for calculating angle and range between sensor nodes. Estimated angels and ranges from a local coordinate, rectified with reference coordinate, and global coordinate is defined for position. In AFN[34], nodes calculate their coordinates.

RSSI is not a better solution as compare to AoA and ToA and TDoA because of environmental conditions.. Most range-based methods suitable for high-density networks and connection disturbance due to larger distance among nodes, make it difficult to calculate distance. The hybrid localization method improves in terms of performance than based methods. In the Hybrid localization method, the time complexity is high and broad calculation is needed to approximate the location.

\section{PERFORMANCE COMPUTATION OF LOCALIZATION ALGORITHM}

The performance computations of localization schemes discuss above are compared in the following table 1:

Table 1: Performance analysis of localization algorithms

\begin{tabular}{|c|c|c|c|c|c|c|c|c|c|c|c|c|c|c|c|}
\hline \multirow[b]{3}{*}{ Fully Range Free } & \multicolumn{6}{|c|}{ Assumption } & \multicolumn{4}{|c|}{ Localization Operations } & \multicolumn{5}{|c|}{ Design } \\
\hline & \multirow[b]{2}{*}{ De } & \multirow[b]{2}{*}{$\mathrm{Nd}$} & \multirow[b]{2}{*}{$\mathrm{Ob}$} & \multirow[b]{2}{*}{ An } & \multirow[b]{2}{*}{$\mathrm{Nm}$} & \multirow[b]{2}{*}{$\mathrm{Ma}$} & \multirow[b]{2}{*}{ Rae } & \multirow[b]{2}{*}{ Rac } & \multirow{2}{*}{ Com } & \multirow{2}{*}{$\mathrm{Lc}$} & \multirow[t]{2}{*}{ Sca } & \multicolumn{3}{|c|}{ Overhead } & \multirow[t]{2}{*}{ Ac } \\
\hline & & & & & & & & & & & & $\mathrm{Cm}$ & $\mathrm{Cp}$ & $\mathrm{He}$ & \\
\hline $\mathrm{CA}$ & Both & $\mathrm{L}$ & $\mathrm{Y}$ & $\mathrm{Y}$ & $\mathrm{N}$ & $\mathrm{N}$ & Conn & Centro & Dist & $2 \mathrm{D}$ & $\mathrm{Y}$ & $\mathrm{L}$ & $\mathrm{L}$ & $\mathrm{L}$ & $\mathrm{L}$ \\
\hline NCA & $\mathrm{R}$ & $\mathrm{L}$ & $\bar{Y}$ & $\mathrm{Y}$ & $\mathrm{N}$ & $\mathrm{N}$ & Conn & Centro & Dist & $3 \mathrm{D}$ & $\mathrm{Y}$ & $\mathrm{L}$ & $\mathrm{M}$ & $\mathrm{L}$ & $\mathrm{L}$ \\
\hline CDV-Hop & $\mathrm{R}$ & $\mathrm{H}$ & - & $\mathrm{Y}$ & $\mathrm{N}$ & $\mathrm{N}$ & Conn & Multi & Dist & $2 \mathrm{D}$ & $\mathrm{N}$ & $\mathrm{H}$ & $\mathrm{L}$ & $\mathrm{L}$ & M \\
\hline ADAL & $\bar{R}$ & $\mathrm{H}$ & $\mathrm{Y}$ & $\mathrm{N}$ & $\mathrm{N}$ & $\mathrm{Y}$ & Conn & Centro & Dist & $2 \mathrm{D}$ & $\mathrm{Y}$ & $\mathrm{L}$ & $\mathrm{L}$ & $\mathrm{L}$ & $\mathrm{M}$ \\
\hline APIT & Both & $\mathrm{H}$ & - & $\mathrm{Y}$ & $\mathrm{N}$ & $\mathrm{N}$ & Conn & Centro & Dist & $2 \mathrm{D}$ & $\mathrm{Y}$ & $\mathrm{H}$ & $\mathrm{L}$ & $\mathrm{L}$ & $\mathrm{M}$ \\
\hline VT-APIT & $\mathrm{R}$ & $\mathrm{M}$ & - & $\mathrm{Y}$ & $\mathrm{N}$ & $\mathrm{N}$ & Conn & Centro & Dist & $3 \mathrm{D}$ & $\mathrm{Y}$ & $\mathrm{H}$ & $\mathrm{L}$ & $\mathrm{L}$ & $\mathrm{M}$ \\
\hline MDS-MAP & $\mathrm{R}$ & $\mathrm{L}$ & - & $\mathrm{N}$ & $\mathrm{N}$ & $\mathrm{N}$ & Conn & Multi & Cent & $2 \mathrm{D}$ & $\mathrm{N}$ & $\mathrm{H}$ & $\mathrm{H}$ & $\mathrm{L}$ & $\mathrm{H}$ \\
\hline IMDS-MAP & $\mathrm{R}$ & $\mathrm{L}$ & - & $\mathrm{N}$ & $\mathrm{N}$ & $\mathrm{Y}$ & Conn & Multi & Dist & $2 \mathrm{D}$ & $\mathrm{Y}$ & $\mathrm{M}$ & $\mathrm{M}$ & $\mathrm{L}$ & $\mathrm{H}$ \\
\hline \multirow[t]{2}{*}{ Fully Range Base } & \multirow[t]{2}{*}{$\mathrm{De}$} & \multirow[t]{2}{*}{$\mathrm{Nd}$} & \multirow[t]{2}{*}{$\mathrm{Ob}$} & \multirow[t]{2}{*}{ An } & \multirow[t]{2}{*}{$\mathrm{Nm}$} & \multirow[t]{2}{*}{$\mathrm{Ma}$} & \multirow[t]{2}{*}{ Rae } & \multirow[t]{2}{*}{ Rac } & \multirow[t]{2}{*}{ Com } & $\mathrm{Lc}$ & Sca & Overl & & & Ac \\
\hline & & & & & & & & & & & & $\mathrm{Cm}$ & $\mathrm{Cp}$ & $\mathrm{He}$ & \\
\hline ILOR & $\mathrm{R}$ & $\mathrm{M}$ & - & $\mathrm{Y}$ & $\mathrm{N}$ & $\mathrm{N}$ & RSSI & MLE & Cent & $2 \mathrm{D}$ & $\mathrm{N}$ & $\mathrm{H}$ & $\mathrm{H}$ & $\mathrm{L}$ & $\mathrm{H}$ \\
\hline COLA & $\mathrm{R}$ & $\mathrm{M}$ & $\mathrm{Y}$ & $\mathrm{Y}$ & $\mathrm{N}$ & $\mathrm{N}$ & RSSI & Trilat & Dist & $3 \mathrm{D}$ & $\mathrm{Y}$ & $\mathrm{L}$ & $\mathrm{M}$ & $\mathrm{L}$ & $\mathrm{M}$ \\
\hline DV-bearing & $\mathrm{R}$ & $\mathrm{H}$ & $\mathrm{Y}$ & $\mathrm{Y}$ & $\mathrm{Y}$ & $\mathrm{N}$ & $\mathrm{AOA}$ & Triang & Dist & $2 \mathrm{D}$ & $\mathrm{Y}$ & $\mathrm{H}$ & $\mathrm{L}$ & $\mathrm{L}$ & $\mathrm{H}$ \\
\hline ALAR & $\mathrm{R}$ & $\mathrm{M}$ & $\mathrm{Y}$ & $\bar{Y}$ & $\mathrm{~N}$ & $\mathrm{~N}$ & $\mathrm{AOA}$ & LSE & Dist & $2 \mathrm{D}$ & $\mathrm{Y}$ & $\mathrm{L}$ & $\mathrm{L}$ & $\mathrm{H}$ & $\mathrm{H}$ \\
\hline MABT & $\mathrm{R}$ & $\mathrm{M}$ & $\mathrm{Y}$ & $\mathrm{N}$ & $\mathrm{N}$ & $\mathrm{Y}$ & TOA & LSE & Dist & $\begin{array}{l}2 \mathrm{D} \\
3 \mathrm{D}\end{array}$ & $\mathrm{Y}$ & $\mathrm{L}$ & $\mathrm{L}$ & $\mathrm{L}$ & $\mathrm{H}$ \\
\hline RSTA & $\mathrm{U}$ & - & $\bar{Y}$ & $\bar{Y}$ & $\mathrm{~N}$ & $\mathrm{~N}$ & TDOA & LSE & Dist & $2 \mathrm{D}$ & $\bar{Y}$ & $\mathrm{~L}$ & $\mathrm{~L}$ & $\mathrm{~L}$ & $\mathrm{H}$ \\
\hline AHLOS & $\mathrm{U}$ & $\mathrm{M}$ & $\mathrm{Y}$ & $\bar{Y}$ & $\mathrm{~N}$ & $\mathrm{~N}$ & TDOA & Multi & Dist & $2 \mathrm{D}$ & $\bar{Y}$ & $\mathrm{~L}$ & $\mathrm{~L}$ & $\mathrm{H}$ & $\mathrm{H}$ \\
\hline$\overline{\mathrm{ABC}}$ & $\mathrm{R}$ & $\mathrm{M}$ & $\mathrm{Y}$ & $\mathrm{N}$ & $\mathrm{N}$ & $\mathrm{N}$ & RSSI & Triang & Dist & $\begin{array}{l}2 \mathrm{D} \\
3 \mathrm{D}\end{array}$ & $\mathrm{N}$ & $\mathrm{L}$ & $\mathrm{L}$ & $\mathrm{L}$ & $\mathrm{L}$ \\
\hline RODL & Both & $\mathrm{M}$ & $\mathrm{Y}$ & $\mathrm{N}$ & $\mathrm{Y}$ & $\mathrm{N}$ & TDOA & Trilat & Dist & $2 \mathrm{D}$ & $\bar{Y}$ & $\mathrm{M}$ & $\mathrm{M}$ & $\mathrm{H}$ & $\mathrm{M}$ \\
\hline Hybrid Range & De & $\mathrm{Nd}$ & $\mathrm{Ob}$ & An & $\mathrm{Nm}$ & $\mathrm{Ma}$ & Rae & Rac & Com & $\mathrm{Lc}$ & Sca & Overl & & & Ac \\
\hline Base & & & & & & & & & & & & $\mathrm{Cm}$ & $\mathrm{Cp}$ & $\mathrm{He}$ & \\
\hline ATPA & $\mathrm{R}$ & $\mathrm{H}$ & $\mathrm{Y}$ & $\mathrm{Y}$ & $\mathrm{Y}$ & $\mathrm{N}$ & $\begin{array}{l}\text { AOA } \\
\text { TOA }\end{array}$ & $\mathrm{BPF}$ & Cent & $2 \mathrm{D}$ & $\mathrm{Y}$ & $\mathrm{M}$ & $\mathrm{M}$ & $\mathrm{H}$ & V.H \\
\hline EATL & Both & $\mathrm{L}$ & $\mathrm{Y}$ & $\mathrm{Y}$ & $\mathrm{N}$ & $\mathrm{N}$ & $\begin{array}{l}\text { AOA } \\
\text { TDOA }\end{array}$ & MLE & Dist & $3 \mathrm{D}$ & $\mathrm{N}$ & $\mathrm{M}$ & $\mathrm{H}$ & $\mathrm{H}$ & V.H \\
\hline FRTL & - & - & $\mathrm{Y}$ & $\mathrm{Y}$ & $\mathrm{N}$ & $\mathrm{N}$ & $\begin{array}{l}\text { TDOA } \\
\text { RSSI }\end{array}$ & LSE & Dist & $2 \mathrm{D}$ & $\mathrm{N}$ & $\mathrm{H}$ & $\mathrm{H}$ & $\mathrm{H}$ & $\mathrm{H}$ \\
\hline HTRL & $\mathrm{U}$ & $\mathrm{L}$ & $\mathrm{Y}$ & $\mathrm{Y}$ & $\mathrm{Y}$ & $\mathrm{N}$ & $\begin{array}{l}\text { TOA } \\
\text { RSSI } \\
\end{array}$ & MLE & Dist & $2 \mathrm{D}$ & $\mathrm{Y}$ & $\mathrm{M}$ & $\mathrm{M}$ & $\mathrm{L}$ & $\mathrm{H}$ \\
\hline SSFL & Both & - & - & $\mathrm{N}$ & $\mathrm{N}$ & $\mathrm{N}$ & $\begin{array}{l}\text { TDOA } \\
\text { AOA }\end{array}$ & Triang & Dist & $\begin{array}{l}2 \mathrm{D} \\
3 \mathrm{D} \\
\end{array}$ & $\mathrm{Y}$ & $\mathrm{M}$ & $\mathrm{H}$ & $\mathrm{H}$ & V.H \\
\hline
\end{tabular}




\begin{tabular}{|c|c|c|c|c|c|c|c|c|c|c|c|c|c|c|c|}
\hline WCA & $\bar{U}$ & - & $\bar{Y}$ & $\mathrm{Y}$ & $\mathrm{N}$ & $\mathrm{N}$ & $\begin{array}{l}\text { RSSI } \\
\text { Conn }\end{array}$ & Centro & Dist & $2 \mathrm{D}$ & $\mathrm{Y}$ & $\mathrm{L}$ & $\mathrm{M}$ & $\mathrm{L}$ & $\mathrm{H}$ \\
\hline RDV-hop & $R$ & $M$ & - & $\mathrm{Y}$ & $\mathrm{N}$ & $\mathrm{N}$ & $\begin{array}{l}\text { RSSI } \\
\text { conn }\end{array}$ & Trilat & Dist & $2 \mathrm{D}$ & $\mathrm{N}$ & $\mathrm{H}$ & $\mathrm{L}$ & $\mathrm{L}$ & $\mathrm{H}$ \\
\hline AFL & $\mathrm{R}$ & $\mathrm{L}$ & - & $\mathrm{N}$ & $\mathrm{Y}$ & $\mathrm{N}$ & $\begin{array}{l}\text { TDOA } \\
\text { conn }\end{array}$ & Trigon & Dist & $\begin{array}{l}2 \mathrm{D} \\
3 \mathrm{D}\end{array}$ & $\mathrm{Y}$ & $\mathrm{H}$ & $\mathrm{M}$ & $\mathrm{H}$ & M \\
\hline TMWC & $\mathrm{U}$ & $\mathrm{M}$ & - & $\mathrm{N}$ & $\mathrm{N}$ & $\mathrm{Y}$ & $\begin{array}{l}\text { RSSI } \\
\text { conn }\end{array}$ & Centro & Dist & $2 \mathrm{D}$ & $\mathrm{Y}$ & $\mathrm{L}$ & $\mathrm{L}$ & M & $\mathrm{H}$ \\
\hline \multirow[t]{2}{*}{ Hybrid Range Free } & \multirow[t]{2}{*}{ De } & \multirow[t]{2}{*}{$\mathrm{Nd}$} & \multirow[t]{2}{*}{$\mathrm{Ob}$} & \multirow[t]{2}{*}{ An } & \multirow[t]{2}{*}{$\mathrm{Nm}$} & \multirow[t]{2}{*}{$\mathrm{Ma}$} & \multirow[t]{2}{*}{ Rae } & \multirow[t]{2}{*}{ Rac } & \multirow[t]{2}{*}{ Com } & \multirow[t]{2}{*}{ Lc } & \multirow[t]{2}{*}{ Sca } & \multicolumn{3}{|c|}{ Overhead } & $\overline{\mathrm{Ac}}$ \\
\hline & & & & & & & & & & & & $\mathrm{Cm}$ & $\mathrm{Cp}$ & $\mathrm{He}$ & \\
\hline IHLA & $\mathrm{R}$ & M & - & $\mathrm{Y}$ & $\mathrm{N}$ & $\mathrm{N}$ & Conn & $\begin{array}{l}\text { Centro } \\
\text { TSE }\end{array}$ & Dist & $2 \mathrm{D}$ & $\mathrm{N}$ & $\mathrm{H}$ & $\mathrm{M}$ & $\mathrm{L}$ & $\mathrm{H}$ \\
\hline
\end{tabular}

\section{A. Communication Cost}

The distribution method demands cooperation between adjacent nodes. However, CDV-Hop[43], based on hop count, needs high communication costs. The centralized method is more efficient and accurate than the distributed method. The cost of centralized is high because data sent back to the base station.

\section{B. Computation Cost}

Energy dissipation data transmission is more than data processing. The processor is $2^{\text {nd }}$ reason to deplete battery life. MDS-MP[36] is a centralized method that is more expensive in terms of forwarding the calculation to processing and solving the matrics. NCA[44] computation cost is more than CA[45]. The computation cost of the hybrid scheme increases with the amount and type of data. A hybrid scheme, either range-based or range-free, needs more computational cost than a full scheme, e.g., Hybrid range free is IHLA[46].

\section{Hardware Cost}

Hardware cost, which includes the measurement equipment, number of nodes, and anchors. In table 1. hardware cost is represented in terms of measurement equipment. The hardware cost of range based ALAR[47] is more than range free CA[48]. Hybrid-range-based scheme EATL[49].and fully-range-based scheme AHLOS[50]. Few hybrid schemes are more expensive because of hardware complexity; two different techniques require two hardware.

\section{Scalability}

When the distance between sensor and receiver increases, the performance of range-based decreases. Wire-less channels become congested in a crowded network, and sophisticated transmission will be required. The centralized is more accurate than the distribution scheme. Centralized undergo scalability, e.g., MDS-MAP[36]. In centralized schemes input data and measured data is proceed and perform operations. Node closest to the base station use more energy. So for dense networks a distributed scheme is always a better solution e.g., IMDS-MAP[51].

\section{CONCLUSION AND FUTURE WORK}

The topic of localization has been discussed in many of the literature so for. In this paper, we try to compare different localization algorithm based on their structure and topology used. All algorithm among studied no one is best everyone have their specification. Range-based hardware cost is high and sensitive to the environment, and range free are fuzzy and easily disturbed by nodes' density. A hybrid scheme is encouraging; in computation, it is affected by execution time. The optimizing algorithm helps to reduce execution time. In the future, many combinations among range measurement and connectivity are highly encouraged in this way of study.

\section{REFERENCES}

[1] S. S. Kulkarni, "TDMA Services for Sensor Networks,"Proc. 24th Int'l. Conf. Distrib. Comp. Sys. Wksps., Mar.2004, pp. 604-09.

[2] W. Ye, J. Heidemann, and D. Estrin, "Medium Access Control with Coordinated Adaptive Sleeping for Wireless Sensor Networks," IEEE/ACM Trans. Net., vol. 12, no. 3, June 2004, pp. 493-506.

[3] A. El-Hoiydi, "Spatial TDMA and CSMA with Preamble Sampling for Low Power Ad Hoc Wireless Sensor Networks," Proc. ISCC 2002, July 2002, pp. 685-92.

[4] Ahmad, T., Li, X. J., \& Seet, B. C. (2017). Parametric loop division for 3D localization in wireless sensor networks. Sensors, 17(7), 1697.

[5] V. Rajendran, K. Obraczka, and J. J. Garcia-Luna-Aceves, "Energy-Efficient, Collision-Free Medium Access Control for Wireless Sensor Networks," Proc. ACM SenSys '03, Los Angeles, CA, Nov. 2003, pp. 181-92.

[6] L. Bao and J. J. Garcia-Luna-Aceves, "A New Approach to Channel Access Scheduling for Ad Hoc Networks," 7th Ann. Int'1. Conf. Mobile Comp. and Net., 2001, pp. 210-21.

[7] Ahmad, Tanveer, Xue Jun Li, and Boon-Chong Seet. "A selfcalibrated centroid localization algorithm for indoor ZigBee WSNs." In 2016 8th IEEE International Conference on Communication Software and Networks (ICCSN), pp. 455461. IEEE, 2016.

[8] Dawadi, B. R., Rawat, D. B., Joshi, S. R., \& Keitsch, M. M. (2020). Towards energy efficiency and green network infrastructure deployment in Nepal using software defined IPv6 network paradigm. The Electronic Journal of Information Systems in Developing Countries, 86(1), e12114.

[9] Ahmad, Tanveer, Xue Jun Li, and Boon-Chong Seet. "3D localization based on parametric loop division and subdivision surfaces for wireless sensor networks." In 2016 25th Wireless and Optical Communication Conference (WOCC), pp. 1-6. IEEE, 2016.

[10] K. Jamieson, H. Balakrishnan, and Y. C. Tay, "Sift: A MAC Protocol for Event-Driven Wireless Sensor Networks," MIT Lab. Comp. Sci., Tech. rep. 894, May 2003, available at http://www.lcs.mit.edu/publications/pubs/pdf/MIT-LCSTR894.pdf

[11] Y. C. Tay, K. Jamieson, and H. Balakrishnan, "CollisionMinimizing CSMA and Its Applications to Wireless Sensor Networks," IEEE JSAC, vol. 22, no. 6, Aug. 2004, pp. 1048-57.

[12] Ahmad, Tanveer, Xue Jun Li, and Boon-Chong Seet. "3D localization using social network analysis for wireless sensor networks." In 2018 IEEE 3rd international conference on communication and information systems (ICCIS), pp. 88-92. IEEE, 2018.

[13] Chen, Hongyang, Pei Huang, Marcelo Martins, Hing Cheung So, and Kaoru Sezaki. "Novel centroid localization algorithm 
for three-dimensional wireless sensor networks." In 2008 4th International Conference on Wireless Communications, Networking and Mobile Computing, pp. 1-4. IEEE, 2008.

[14] Chen, Kai, Zhong-hua Wang, Mei Lin, and Min Yu. "An improved DV-Hop localization algorithm for wireless sensor networks." (2010): 255-259.

[15] zeng Wang, Ji, and Hongxu Jin. "Improvement on APIT localization algorithms for wireless sensor networks." In 2009 International Conference on Networks Security, Wireless Communications and Trusted Computing, vol. 1, pp. 719-723. IEEE, 2009.

[16] Ahmad, Tanveer, Xue Jun Li, and Boon-Chong Seet. "Noise Reduction Scheme for Parametric Loop Division 3D Wireless Localization Algorithm Based on Extended Kalman Filtering." Journal of Sensor and Actuator Networks 8, no. 2 (2019): 24.

[17] Ahmad, Tanveer, Xue Jun Li, and Boon-Chong Seet. "FuzzyLogic Based Localization for Mobile Sensor Networks." In 2019 2nd International Conference on Communication, Computing and Digital systems (CCODE), pp. 43-47. IEEE, 2019.

[18] G. Lu, B. Krishnamachari, and C. S. Raghavendra, "An Adaptive Energy-Efficient and Low-Latency MAC for Data Gathering in Wireless Sensor Networks," Proc. 18th Int'l. Parallel and Distrib. Processing Symp., Apr. 2004, p. 224.

[19] Guerrero, Esteban, H. G. Xiong, Qiang Gao, Gabriel Cova, Ricardo Ricardo, and J. Estévez. "ADAL: A distributed range-free localization algorithm based on a mobile beacon for wireless sensor networks." In 2009 International Conference on Ultra Modern Telecommunications \& Workshops, pp. 1-7. IEEE, 2009.

[20] Ahmad, Tanveer, Xue Jun Li, Boon-Chong Seet, and JuanCarlos Cano. "Social Network Analysis Based Localization Technique with Clustered Closeness Centrality for 3D Wireless Sensor Networks." Electronics 9, no. 5 (2020): 738.

[21] hakila, R., and B. Paramasivan. "An improved range based localization using Whale Optimization Algorithm in underwater wireless sensor network." Journal of Ambient Intelligence and Humanized Computing (2020): 1-11.

[22] Ahmad, T. (2019). 3D Localization Techniques for Wireless Sensor Networks (Doctoral dissertation, Auckland University of Technology).

[23] R. Niu and P. Varshney, "Target location estimation in wireless sensor networks using binary data," in Proceedings of the 38th International Conference on Information Sciences and Systems, pp. 17-19, Princeton, NJ, USA, March 2004.

[24] Ahmad, T. (2019). 3D Localization Techniques for Wireless Sensor Networks (Doctoral dissertation, Auckland University of Technology).

[25] M. P. Michaelides and C. G. Panayiotou, "SNAP: fault tolerant event location estimation in sensor networks using binary data," IEEE Transactions on Computers, vol. 58, no. 9, pp. 1185- 1197, 2009.

[26] Ahmad, T., Li, X. J., Wenchao, J., \& Ghaffar, A. (2020, September). Frugal Sensing: A Novel approach of Mobile Sensor Network Localization based on Fuzzy-Logic. In Proceedings of the ACM MobiArch 2020 The 15th Workshop on Mobility in the Evolving Internet Architecture (pp. 8-15).

[27] K. Lu, X. Xiang, D. Zhang, R. Mao, and Y. Feng, "Localization algorithm based on maximum a posteriori in wireless sensor networks," International Journal of Distributed Sensor Networks, vol. 2012, Article ID 260302, 7 pages, 2012.

[28] L. Cheng, C. D. Wu, Y. Z. Zhang, and Y. Wang, "Indoor robot localization based on wireless sensor networks," IEEE Transactions on Consumer Electronics, vol. 57, no. 3, pp. 1099-1104, 2011.

[29] Y. Wang, X. Wang, D. Wang, and D. P. Agrawal, "Rangefree localization using expected hop progress in wireless sensor networks," IEEE Transactions on Parallel and Distributed Systems, vol. 20, no. 10, pp. 1540-1552, 2009.

[30] H. Xu, Y. Tu, W. Xiao, Y. Mao, and T. Shen, "An archimedes curve-based mobile anchor node localization algorithm in wireless sensor networks," in Proceedings of the 8th World Congress on Intelligent Control and Automation (WCICA '10), pp. 6993-6997, Jinan, China, July 2010.

[31] J. Lee, W. Chung, and E. Kim, "Robust DV-Hop algorithm for localization in wireless sensor network," in Proceedings of the International Conference on Control, Automation and Systems, pp. 2506-2509, Gyeonggi-do, South Korea, October 2010.

[32] Ware, R.; Lad, F. Approximating the Distribution for Sum of Product of Normal Variables; Research report; the Mathematics and Statistics department at Canterbury University: Christchurch, New Zealand, 2003.

[33] Wen, C.-Y.; Hsiao, Y.-C. Decentralized anchor-free localization for wireless ad-hoc sensor networks. In Proceedings of IEEE International Conference on Systems, Man, and Cybernetics, Singapore, October 2008; pp. 2777 2785.

[34] Roberts, G.; Gelman, A.; Gilks, W. Weak Convergence and Optimal Scaling of Random Walk Metropolis Algorithms. Technical Report; University of Cambridge: Cambridge, UK, 1994.

[35] Chan, Fu and Chih-yu "Adaptive AoA/ToA localziation using fuzzy particle for mobile WSNs" in 2011 IEEE $73^{\text {rd }}$ VTC Spring 2011.

[36] Liu, Q.; Ihler, A. T.; Smyth, P. Particle filtered MCMC-MLE with connections to contrastive divergence. In Proceedings of the 27 th International Conference on Machine Learning, Haifa, Israel, June 2010; pp. 1-8.

[37] Canovas, J. P.; LeBlanc, K.; Saffiotti, A. Robust multi-robot object localisation using fuzzy logic. In Proceedings of 2004 Int. Robocup Symposium, Lisbon, Portugal, July 2004; pp. 247-261.

[38] Liu, C.; Wu, K.; He, T. Sensor localization with ring overlapping based on comparison of received signal strength indicator. In Proceedings of IEEE Mobile Ad-hoc and Sensor Systems (MASS'04), Fort Lauderdale, FL, USA, October 2004; pp. 516-518.

[39] Doucet, A.; de Freitas, N.; Gordon, N. Sequential Monte Carlo Methods in Practice; Springer-Verlag: New York, NY, USA, 2001.

[40] Chib, S.; Greenberg, E. Understanding the MetropolisHastings algorithm. Amer. Statist. 1995, 49, 327-335.

[41] Chintalapudi, K.K.; Dhariwal, A.; Govindan, R.; Sukhatme, G. Ad-hoc localization using ranging and sectoring. In Proceedings of INFOCOM, Hong Kong, China, 7-11 March 2004; pp. 2662-2672.

[42] Ihler, A.T.; Fisher, J.W.; Moses, R.L.; Willsky, A.S Nonparametric belief propagation for self-localization of sensor networks. IEEE J. Sel. Areas Commun. 2005,

[43] Wu, Jiawei, Jinming Yu, Aijun Ou, Yiming Wu, and Wujun Xu. "RCDV-Hop Localization Algorithm for WSN." In 2012 8th International Conference on Wireless Communications, Networking and Mobile Computing, pp. 1-4. IEEE, 2012.

[44] Chen, W.; Mei, T.; Meng, M. Q.; Liang, H.; Liu, Y.; Li, Y.; $\mathrm{Li}$, S. Localization algorithm based on a spring model (LASM) for large scale wireless sensor networks. Sensors 2008, 3, 1797-1818.

[45] Savvides, A.; Park, H.; Srivastava, M. The bits and flops of the N-hop multilateration primitive for node localization problems. In Proceedings of the First ACM International Workshop on Sensor Networks and Applications, Atlanta, GA, USA, September 2002; pp. 112-121.

[46] Bala, M., \& Kumar, M. (2019). An Energy-Efficient routing protocol based on TABU-Genetic Strategy in Wireless Sensor Network. International Journal of Advanced Networking and Applications, 10(6), 4099-4104 